\title{
The Effects of Six Weeks of Balance and Strength Training on Measures of Dynamic Balance of Older Adults
}

\author{
Christopher J. Knerl1 ${ }^{1}$, Petra B. Schuler ${ }^{1}$, Lemuel W. Taylor ${ }^{2}$, Ludmila M. Cosio-Lima ${ }^{1}$, Karla A. \\ Caillouet $^{1}$ \\ ${ }^{1}$ Department of Health, Leisure and Exercise Science, The University of West Florida, \\ Pensacola, FL, USA
${ }^{2}$ Department of Exercise Science and Sports Science, University of Mary Hardin-Baylor, Belton, TX, USA

\begin{abstract}
This study examined the effects of six weeks of strength training, balance training, and a combination of both on dynamic balance in older adults. Fifty-one seniors (17 males and 34 females) between the ages of 60 and 93 years $(73.2+7.9 \mathrm{yrs})$ participated. Four groups, three treatment groups [strength training (ST), balance training (BT), and a group which combined strength and balance training (SAB)] and a control group (Control), were tested on measures of dynamic balance, strength, and flexibility before (pre-test) and after six weeks of exercise training (post-test). Measures of dynamic balance included the 8-Foot-Up and Go Test and the combined score on the Fullerton Advanced Balance Scale (FAB-Scale). Lower and upper body strength and flexibility were assessed using components of the Senior Fitness Test (chair stand, arm curl, sit and reach, and back scratch test). A repeated measure MANCOVA showed no significant differences between groups for measures of dynamic balance. Repeated measure ANOVA's with Post Hoc comparisons indicated upper body strength and lower body flexibility increased significantly $(\mathrm{p}<0.05)$ in the $\mathrm{ST}$ and $\mathrm{SAB}$ group while upper body flexibility decreased significantly $(\mathrm{p}<$ 0.05 ) in the ST group compared to all other groups. Although all treatment groups exhibited a trend for improvement, results suggest six weeks of exercise training, regardless of type, was not sufficient to elicit significant changes in dynamic balance in the present sample of older adults.
\end{abstract}

(C) 2009 Californian Journal of Health Promotion. All rights reserved.

Keywords: Elderly, older adults, exercise, fall prevention.

\section{Introduction}

It has been estimated that by the year 2020, the total annual cost of injuries related to falls in elderly individuals will reach more than $\$ 32.4$ billion (Merck Institute of Aging \& Health, 2004; Stevens \& Sogolow, 2008; Stevens, Corso, Finkelstein, \& Miller, 2006). Unintentional injuries have been ranked among the top five causes of death in the United States and falls have been identified as a significant cause of such injuries (Holmes, 1999). Lifethreatening or not, falls in the elderly often result in a loss of mobility and independence that generally results in a decrease in the quality of life (Brouwer, Musselmann, \& Culham, 2004). Falls in the elderly, either directly or indirectly, affect the vast majority of Americans. Falls result, for instance, in an increased health and social service cost because of injury, inability to maintain a safe environment, and dependence in basic functional activities (Steadman, Donaldson, \& Kalra, 2003; Steven, Corso, Finkelstein, \& Miller, 2006). It is obvious that falls, which directly affect one-third of all individuals age 65 and older, and which indirectly affect the financial health of all Americans, are a serious issue that needs further attention (Campbell \& Robertson, 2007; 
Holmes, 1999; Huang, Liu, Huang, \& Kernohan,

2010; Rose, 2008).

As they age, elderly individuals experience a decrease in strength and balance that has been listed as a risk factor for falls (Bird, Hill, Ball, \& Williams, 2009; Piirtola \& Era, 2006; Rubenstein, 2006; Stevens, 2005). For example, Rubenstein (2006) reported that older individuals with reduced leg strength exhibited 4.9 times the risk of falling compared to people with normal leg strength. Balance is defined as the ability to maintain an upright posture during static and dynamic tasks (Benjuva, Melzer, \& Kaplanski, 2004). Maintaining balance requires complex interactions between peripheral and central factors such as vision, somatosensation, vestibular sensation, motor output, and musculature (Dodd, Taylor, \& Bradley, 2004). All of these factors decline with advancing age. A review of the literature shows that many treatments can help reduce the risk for falls, including strength training, balance training, home modification, and vision improvements (Barnett, Smith, Lord, Williams, \& Baumand, 2003; Campbell \& Robertson, 2007; Cumming et al., 1999; Frick, Kung, Parrish, \& Narrett, 2010; Huang, Liu, Huang, \& Kernohan, 2010; Li, Harmer, Fisher, \& McAuley, 2004; Province et al., 1995; Pynoos, 2005; Rose, 2005; Stevens, 2005; The National Council on Aging, 2005; Tinetti, 1988; Wolf et al., 2003). With one expectation (Campbell \& Robertson, 2007), authors agree that multifactorial interventions targeting both intrinsic (aging, depression, hypertension, diabetes, poor gait, taking more than four medications) and extrinsic (fear of falling, footwear, inside and outside environmental hazards) risk factors for falls are most effective in decreasing the occurrence of falls. Campbell and Robertson (2010) completed a meta-analysis of 90 fall risk reduction trials in community-living older adults. Based on their findings, the authors concur that multifactorial interventions are highly effective for individual patients; however, for community programs, targeted single interventions appear equally as effective and perhaps even more acceptable and cost effective.
With respect to single interventions, exercise as a stand-alone intervention has been studied quite extensively over the past $20-25$ years and has generally been shown to be effective in reducing fall risk and/or fall incidence rates (for comprehensive reviews see Costello \& Edelstein, 2008; Rose, 2005, 2008). Exercise programs studied consisted of single modes of exercise (e.g. resistance training, balance training, Tai Chi, or flexibility training) (Bird, Ball, \& Williams, 2009; Kim \& Lockhart, 2010; Woo, Hong, Lau, \& Lynn, 2007), or multicomponent exercise programs incorporating aerobic endurance, flexibility, strength and/or balance training (Barnett, Smith, Lord, Williams, \& Baumand, 2003; Lord, et al., 2003; Province et al., 1995). Even though the evidence supporting exercise as an effective intervention is strong, important questions remain, including what type of exercise is most effective and what dose is needed to elicit positive changes (Costello \& Edelstein, 2008, Rose, 2008; Tinetti, 1994). For example, a minimum duration of 12 weeks for an effective exercise intervention to reduce the risk of falling has been suggested (Costello \& Edelstain, 2008); however, improvements in balance, proprioception, lower leg strength, and reaction time have been reported following only six weeks of exercise training in older individuals with type-2 diabetes (Morrison, Colberg, Marioano, Parson, \& Vinik, 2010); these exercises included balance, flexibility, and strength training. Similar discrepancies persist with respect to what type of exercise is most beneficial. For example, Woo and colleagues (2007) reported no changes in balance, flexibility, or the number of falls after 12 months of Tai Chi and resistance exercise in a sample of community-dwelling older adults; on the other hand, Bird, Ball, \& Williams (2009) showed significant improvements in balance performance after 16 weeks of resistance and flexibility training.

The purpose of the present study was twofold: (1) to compare the effectiveness of strength training (ST), balance training (BT), a 
combination of strength and balance training (SAB), and no training (Control), on dynamic balance in the elderly; and (2) to determine if six weeks of training would be enough to elicit positive changes in dynamic balance in this group of community-dwelling older adults. Dynamic balance is the ability to maintain equilibrium during movement. It was hypothesized that there would be a significant difference between groups (ST, BT, SAB, and Control) on the time it takes to complete the 8Foot-Up and Go Test and the combined score on the Fullerton Advanced Balance Scale (FABScale) after six weeks of exercise training. The 8-Foot-Up and Go Test, part of the Senior Fitness Test, is a strong measure of dynamic balance and may be the most effective single test for measuring dynamic balance in the elderly (Rikli, 1999). The FAB-Scale has been shown to be a valid and reliable assessment tool suitable for functionally independent communitydwelling older adults (Rose, Lucchese, \& Wiersma, 2006).

\section{Methods}

\section{Participants}

Initially, 54 elderly individuals (18 males; 36 females) were recruited from the local community to participate in this study; fifty-one individuals (17 males; 34 females) completed the study. One male participant had to be omitted from the control group because, due to time constraints, he was unable to attend the post-assessment session; two female participants were eliminated from the strength training group because they did not meet the $75 \%$ attendance requirement. Participants in the exercise groups (ST, BT, and SAB) were recruited from ongoing programs at the University; hence, they selfselected their treatment group. The control group was recruited from two local senior centers (see Table 1 for Descriptive Statistics). All participants signed an informed consent approved by the University's Institutional Review Board. Inclusion criteria were as follows: participants had to be 60 years of age or older, able to walk without assistance, attend $75 \%$ of the training sessions, attend both the preand post-test assessment sessions, and obtain medical clearance from a physician. The control group was instructed not to participate in any structured exercise training programs throughout the duration of the study.

\section{Protocol}

Study procedures were explained in an informal meeting prior to the beginning of the study. All participants' questions were answered at this point; after that, participants signed an informed consent and medical clearance form. The study had three phases: pre-test assessments (one day), exercise training phase (six weeks), and post-test assessments (one day).

\section{Pre-Test Assessment Protocol}

After arriving at the University of West Florida's Health, Leisure and Sports Facility, participants were asked to rest (quiet sitting) for five minutes, after which resting heart rate, blood pressure, height, and weight were measured. All participants then performed a brief warm-up consisting of a short walk on the indoor track (1/4 mile) followed by simple stretches for upper and lower body (shoulder, chest, back, hamstrings, and quadriceps). Participants were then asked to complete the Senior Fitness Test and the FAB-Scale. The FAB-Scale uses a ten item approach that measures multiple dimensions of balance. The FAB-Scale includes: standing with feet together and eyes closed for 30 seconds, reaching forward to retrieve a pencil ten inches away, turning in a circle to the left and the right, stepping up and over a six-inch bench, tandem walk for ten steps, standing on one leg with eyes open for 20 seconds, standing on foam with eyes closed for 20 seconds, two-footed jump for distance, walk with head turns, and an unexpected backward release to measure reactive postural control. Each test is scored on a four point scale, with four being a perfect score and zero assigned if the participant is unable or unwilling to attempt any aspect of the tasks. A detailed rubric to score each test item was provided by the authors in their original publication (Rose, Lucchese, \& Wiersma, 2006). For example, if a participant assumes the correct standing position in test one (standing with feet together and eyes closed for 30 seconds) but can only close the eyes for 20 seconds, his/her performance would be scored as two; if the eyes 
were closed for less than ten seconds in the correct standing position the individual would receive a score of one.

The Senior Fitness Test was used to assess each individual's upper and lower body strength, balance, and flexibility. The Senior Fitness Test includes a 30-second chair stand, arm curl, chair sit and reach, back scratch, and the 8-Foot-Up and Go Test. For example, for the 8-Foot-Up and Go Test, the participant is seated in a chair. On the signal "go," the participant gets up, walks as quickly as possible around a cone placed eight feet away, and returns to the chair. The participant is scored based on the time it takes him or her to complete the task. For the back scratch test, the participant is asked to place the preferred hand behind the same-side shoulder, to place the other hand behind the back as well, and then to touch or overlap the extended middle fingers of both hands. Performance is scored by measuring the distance between the fingertips of both hands. A negative score is given if the fingertips do not overlap. For a detailed description of each test item please see Rikli \& Jones, (2001).

The Senior Fitness Test and the FAB-Scale are easy to administer and safe to perform for older adults. Furthermore, both tests have been shown to have high reliability and validity (Rikli \& Jones, 2001; Rose, Lucchese, \& Wiersma, 2006). All tests were administered and scored by a group of trained instructors selected from students who excelled in a senior level course specializing in exercise testing and prescription for older adults. All students were required to take a practical examination, which consisted of administering and scoring the Senior Fitness Test and the FAB-Scale; only students with perfect practical scores were selected as test administrators for the study. A total of 12 students were selected to administer all pre- and post-test assessments.

\section{Exercise Training Phase}

Upon completion of the pre-test assessments, participants joined their respective groups (ST, $\mathrm{BT}$, or SAB). Control group participants were instructed not to engage in any structured exercise program throughout the six week training phase, although the control group's activity level was not monitored. Upon completion of the six week training phase all control group participants affirmed their strict adherence to these instructions. Participants in the ST and BT groups met twice per week (Tuesday/Thursday) for one hour each day for six weeks (a total of 12 hours of training). The exercises for the ST group included abdominal crunches, chest press, seated row, shoulder lateral raises, leg press, knee extension, hip adduction/abduction, calf rises, knee curls, biceps curls, tricep push downs, lat pull downs, and back extensions. All exercises were performed on Nautilus Nitro Plus fitness equipment, under the supervision of a student trainer. All training sessions included a brief warm-up and cool-down. The program was designed to strengthen all major muscle groups with six upper body, six lower body, and two core strengthening exercises (Baechle \& Earle, 2008; Westcott \& Baechle, 1999). .

Initial loads were determined by identifying a load that represented approximately $75 \%$ of the individual's estimated One Repetition Maximum (1-RM). Seventy-five percent of an elderly individual's 1-RM corresponds with the amount of load that individual can comfortably lift 8-12 times (Westcott, 2005). The workload was increased by first increasing repetitions, followed by an increase in load. Once participants were able to comfortably perform one set of 8-10 repetitions, they increased to one set of 10-12 repetitions while maintaining the same load. When participants were comfortable with one set of 10-12 repetitions, load was added and repetitions were lowered to 8-10. After two weeks, if participants were comfortable with the loads, two sets were completed while using the same repetition progression. Rest time between sets was 1-2 minutes or as much time as was needed to move from exercise to exercise. Modifications were made to individuals' exercises depending on the participants' needs. Loads and number of repetitions were recorded in a strength training log sheet during each exercise session. The upkeep of these log books allowed for consistency between sessions as 
well as a means for attendance verification. Participants were excluded from the study if their attendance rate dropped below $75 \%$.

The BT group followed the FallProof ${ }^{\mathrm{TM}}$ program. The FallProof ${ }^{\mathrm{TM}}$ program is a structured and progressive exercise program designed to improve balance and mobility. FallProof ${ }^{\mathrm{TM}}$ was recognized by the National Council on Aging as one of the seven exemplary model programs in the United States promoting healthy aging and improved quality of life (The National Council on Aging, 2005). Each session had the following components: multisensory training exercises, hand-eye coordination exercises, gait pattern enhancement and variation training exercises, strength and flexibility exercises, and a brief warm-up and cool-down (a detailed description of the program including sample training sessions are provided by Rose, 2003). The strength component of the FallProof ${ }^{\mathrm{TM}}$ program was very brief and consisted of Thera-Band work only. This was not a major focus of the BT program. Participants in the SAB training group met twice a week for both the strength training sessions as well as the balance training sessions for a total of four hours per week for six weeks. These strength training and balance training sessions were identical to the ST and BT group sessions.

\section{Post-Test Assessment Protocol}

Upon completion of the six week training phase, each participant completed the post-test assessments. The post-test assessment protocol included a brief warm-up, 8-Foot-Up and Go Test, FAB-Scale, and a cool-down. The tests were administered in the same manner and by the same evaluators who directed the pre-test assessments.

Table 1. Means and Standard Deviations $(M \pm S D)$ for descriptive variables, Senior Fitness Test- and FAB-Scale Total Scores, and p-values for group comparisons (Pre-Test Assessments)

\begin{tabular}{|c|c|c|c|c|c|}
\hline & $\mathrm{ST}(\mathrm{N}=9)$ & BT $(\mathrm{N}=11)$ & SAB $(\mathbf{N}=18)$ & $C(\mathrm{~N}=13)$ & $p$-value \\
\hline Age (yrs) & $68.40 \pm 7.93$ & $77.25 \pm 7.44$ & $73.42 \pm 8.70$ & $72.93 \pm 6.08$ & 0.077 \\
\hline Height (m) & $1.70 \pm 0.09$ & $1.62 \pm 0.07$ & $1.67 \pm 0.11$ & $1.64 \pm 0.08$ & 0.184 \\
\hline Weight (kg) & $80.61 \pm 21.48$ & $71.22 \pm 13.80$ & $71.06 \pm 12.90$ & $81.16 \pm 16.84$ & 0.183 \\
\hline $\begin{array}{l}\text { Arm } \\
\text { (reps) }\end{array}$ & $13.10 \pm 3.82$ & $10.45 \pm 3.31$ & $12.36 \pm 3.54$ & $15.14 \pm 5.41$ & 0.028 \\
\hline $\begin{array}{l}\text { Sit and } \\
\text { Reach (in) }\end{array}$ & $0.83 \pm 2.80$ & $-0.56 \pm 2.83$ & $1.18 \pm 4.09$ & $-2.03 \pm 3.63$ & 0.039 \\
\hline $\begin{array}{l}\text { Back Scratch } \\
\text { (in) }\end{array}$ & $-0.50 \pm 3.71$ & $-4.29 \pm 4.33$ & $-1.40 \pm 3.72$ & $-5.32 \pm 3.20$ & 0.004 \\
\hline $\begin{array}{l}\text { Chair Stand } \\
\text { (reps) }\end{array}$ & $11.90 \pm 3.35$ & $10.45 \pm 3.82$ & $10.47 \pm 4.13$ & $11.78 \pm 2.20$ & 0.459 \\
\hline $\begin{array}{l}\text { 8-Foot-Up- } \\
\text { Go (s) }\end{array}$ & $6.06 \pm 1.63$ & $6.99 \pm 1.46$ & $6.49 \pm 1.68$ & $6.00 \pm 1.53$ & 0.299 \\
\hline FAB-Total & $30.50 \pm 5.10$ & $27.91 \pm 4.44$ & $28.47 \pm 6.20$ & $29.85 \pm 4.63$ & 0.622 \\
\hline
\end{tabular}

\section{Statistical Analysis}

Means and standard deviations were calculated for all descriptive variables as well as preassessment Senior Fitness Test and FAB-Scale scores (see Table 1). A MANOVA was used to test for group differences for all pre-test assessments (see Table 1 for significant group differences). The study's hypothesis was tested with a $2 \times 2$ repeated measures MANCOVA [2 variables (8-Foot-Up and Go/FAB-Scale total 
score) by time (pre- and post-test measures)] with three pre-test measures (arm curl, sit and reach, back scratch) used as covariates (see Table 2). A repeated measures ANOVA was used to assess changes for each of the remaining Senior Fitness Test variables (chair stand, arm curl, sit and reach test, and back scratch test);
Tukey's Least Significant Difference (LSD) was used for pairwise comparisons (see Table 3). All statistical procedures were performed using SPSS 15.0 software (Chicago, IL) and a probability level of $\leq 0.05$ was adopted throughout.

Table 2. Means and Standard Deviations $(\mathrm{M} \pm \mathrm{SD})$ and number of participants per group (N)

$\begin{array}{llrrllr}\text { Pre-Test Assessment } & \text { Group } & \text { M } \pm \text { SD }^{\mathbf{a}} & \mathbf{N} & \begin{array}{l}\text { Post-Test } \\ \text { Assessment }\end{array} & \text { Group } & \mathbf{M} \pm \mathbf{S D}^{\mathbf{a}} \\ \text { 8-Foot-Up and Go } & \text { ST } & 6.17 \pm 1.67 & 18 & \text { 8-Foot-Up and Go } & \text { ST } & 5.12 \pm 0.86 \\ & \text { BT } & 7.21 \pm 1.44 & 9 & & \text { BT } & 6.83 \pm 1.95 \\ & \text { SAB } & 6.50 \pm 1.70 & 11 & & \text { SAB } & 6.23 \pm 1.55 \\ & \text { C } & 6.02 \pm 1.53 & 13 & & \text { C } & 6.05 \pm 1.45 \\ \text { FAB-Total Score } & \text { ST } & 30.50 \pm 5.10 & 18 & \text { FAB-Total Score } & \text { ST } & 32.00 \pm 4.84 \\ & \text { BT } & 27.91 \pm 4.44 & 9 & & \text { BT } & 29.18 \pm 4.19 \\ & \text { SAB } & 28.47 \pm 6.20 & 11 & & \text { SAB } & 31.94 \pm 5.25 \\ & \text { C } & 29.85 \pm 4.63 & 13 & \text { C } & 29.92 \pm 4.21\end{array}$

a Covariates appearing in the model are evaluated at the following values: arm curl $=12.74$, sit and reach $=-0.78$, back scratch $=$ $-2.98$

\section{Results}

Pre-test comparisons (MANOVA) revealed a significant multivariate effect for group $($ Wilks'Lamda $=0.57, \mathrm{~F}(15 / 130)=1.93, \mathrm{p}<0$. $05)$; significant between-subjects effects for group indicated differences in upper body strength $(\mathrm{F}(3 / 47)=3.28, \mathrm{p}<0.05)$, lower-body flexibility $(\mathrm{F}(3 / 47)=3.00, \mathrm{p}<0.05)$, and upperbody flexibility $(\mathrm{F}(3 / 47)=4.96, \mathrm{p}<0.05)$. Post hoc analyses suggested that the control group had significantly greater upper body strength compared to the BT group; the control group also scored significantly below the ST and SAB group on lower and upper body flexibility measures (see Table 1).

It was hypothesized that there would be a significant difference between groups (ST, BT, $\mathrm{SAB}$, and Control) with respect to the time it took to complete the 8-Foot-Up and Go Test and the combined score on the Fullerton Advanced Balance Scale (FAB-Scale) after six weeks of exercise training. The results of the repeated measures MANCOVA did not support this hypothesis. Both the result of the multivariate tests (Wilks'Lamda $=0.97, \mathrm{~F}(3 / 44)=1.93, \mathrm{p}>$ $0.05)$ as well as the between-subjects effect for group were not significant $(\mathrm{F}(3 / 44)=0.12, \mathrm{p}>$ 0.05 ) (see Table 2 for means and standard deviations). When controlling for pre-test group differences, no significant differences between groups were found for either measure of dynamic balance after six weeks of exercise training.

A repeated measures ANOVA conducted for each of the remaining measures of the Senior Fitness Test showed significant differences between groups for upper body strength $(\mathrm{F}(3 / 47)$ $=3.29, \mathrm{p}<0.05)$, lower body flexibility $(\mathrm{F}(3 / 47)=3.17, \mathrm{p}<0.05)$ and upper body flexibility $(\mathrm{F}(3 / 47)=4.13, \mathrm{p}<0.05)$. Post Hoc analyses indicated that upper body strength and lower body flexibility increased significantly in the strength training (ST) group and the combined group (SAB) compared to the control group. On the other hand, upper body flexibility decreased significantly in the ST group compared to all other groups. No significant changes (group as well as time) were observed for lower body strength (see Table 3 ). 


\section{Discussion}

Two recent reviews (Costello \& Edelstein, 2008; Rose, 2008) on the effectiveness of various intervention strategies to prevent falls in older adults have identified several questions to be addressed in future research when considering exercise as a means for reducing falls. According to both reviews, it is currently unclear what type of exercise is most effective, what dose is needed to elicit positive changes, which population should be targeted, and which fall related outcome measures are most effective. The present study attempted to answer two of these questions: (1) what type of program is most effective; and (2) whether six weeks of training is sufficient to elicit positive changes in the selected measures of dynamic balance for the sample of community-dwelling older adults. It was hypothesized that after six weeks of exercise training there would be a significant difference between groups on the time it takes to complete the 8-Foot-Up and Go Test and the combined score on the Fullerton Advanced Balance Scale (FAB-Scale). The findings of the present study did not support the hypothesis. Even though all treatment groups showed a trend toward improvement, while the control group remained unchanged, there were no significant differences between groups for either measure of dynamic balance after six weeks of exercise.

Table 3. Means and Standard Deviations $(\mathrm{M} \pm \mathrm{SD}$ ) for the remaining Senior Fitness Variables (Pre- and Post-Test Assessments) with $\mathrm{p}$ values for group comparisons

\begin{tabular}{|c|c|c|c|c|c|}
\hline & $\mathrm{ST}(\mathrm{N}=9)$ & BT $(\mathrm{N}=11)$ & SAB $(\mathbf{N}=18)$ & $C(N=13)$ & $p$-value \\
\hline \multirow{2}{*}{$\begin{array}{l}\text { Pre Arm Curls (reps) } \\
\text { Post Arm Curls }\end{array}$} & $13.10 \pm 3.82$ & $10.45 \pm 3.31$ & $12.36 \pm 3.54$ & $15.14 \pm 5.41$ & \\
\hline & $20.77 \pm 5.56^{\mathrm{a}}$ & $13.63 \pm 4.27$ & $17.94 \pm 3.65^{\mathrm{a}}$ & $17.23 \pm 6.78$ & 0.028 \\
\hline $\begin{array}{l}\text { Pre Sit and Reach } \\
\text { (in) }\end{array}$ & $0.83 \pm 2.80$ & $-0.56 \pm 2.83$ & $1.18 \pm 4.09$ & $-2.03 \pm 3.63$ & \\
\hline Post Sit and Reach & $0.97 \pm 2.72^{\mathrm{a}}$ & $-0.27 \pm 4.36$ & $1.69 \pm 4.86^{\mathrm{a}}$ & $-2.28 \pm 3.64$ & 0.033 \\
\hline $\begin{array}{l}\text { Pre Back Scratch } \\
\text { (in) }\end{array}$ & $-0.50 \pm 3.71$ & $-4.29 \pm 4.33$ & $-1.40 \pm 3.72$ & $-5.32 \pm 3.20$ & \\
\hline Post Back Scratch & $-1.75 \pm 3.78^{b}$ & $-4.75 \pm 5.99$ & $-1.61 \pm 3.83$ & $-5.69 \pm 3.72$ & 0.011 \\
\hline $\begin{array}{l}\text { Pre Chair Stand } \\
\text { (reps) }\end{array}$ & $11.90 \pm 3.35$ & $10.45 \pm 3.82$ & $10.47 \pm 4.13$ & $11.78 \pm 2.20$ & \\
\hline Post Chair Stand & $14.55 \pm 3.24$ & $11.63 \pm 4.17$ & $13.16 \pm 5.10$ & $12.00 \pm 2.76$ & 0.515 \\
\hline
\end{tabular}

a ST and SAB group are significantly different at the $\mathrm{p}<0.05$ level from the control group b ST group is different from all other groups at the $\mathrm{p}<0.05$ level

While the present study failed to provide evidence to support one exercise intervention over another, the findings may suggest that six weeks of training, regardless of the type of exercise performed, was not enough of a stimulus to elicit significant changes in the dynamic balance of community-dwelling older adults. Contrary to the present findings, Henwood \& Taaffe (2006) showed that as little as eight weeks of high-velocity strength training produced significant improvements in a five repetition Sit-to-Stand (STS) test in a sample of community-dwelling older adults (Henwood \& Taaffe, 2006). The STS has been correlated to falls and balance in older adults (Henwood \&
Taaffe, 2006). Eight weeks of resistance and balance training were also reported to be a sufficient stimulus to significantly reduce the likelihood of slips and improve the recovery from slips (Kim \& Lockhart, 2010) in a similar sample of community-dwelling older adults. Improvements in balance, proprioception, lower leg strength, and reaction time have been demonstrated following as little as six weeks of exercise training (balance, flexibility, and strength training) in older individuals with type2 diabetes (Morrison, Colberg, Marioano, Parson, \& Vinik, 2010). However, since older individuals with type-2 diabetes often present greater impairments in posture and gait resulting 
in a greater risk of falls (Maurer, Burcham, \& Cheng, 2005), direct comparisons cannot be made. Older adults often face barriers to exercise such as transport, time, access, or cost and it is therefore important to identify a minimal threshold with respect to program duration. Unfortunately, the limitations of the present study, including the small sample size and the lack of randomization between treatment groups, make it difficult to identify such a threshold. Costello and Edelstein (2008), in their review of literature, suggested a minimum duration of 12 weeks for an effective exercise intervention program to reduce the risk of falls.

Costello \& Edelstein (2008) have also suggested that one reason for the lack of treatment effects in studies investigating the effect of exercise on fall risk reduction as a stand-alone intervention might be characteristics of the population studied. That is, participants were at either too high or too low a risk for falls to benefit from the intervention. This was not the case for the participants in the present study; they were relatively healthy, community-dwelling, independent-living older adults. Their performance on both measures of balance (8Foot-Up and Go Test and total score on the FAB-Scale) was in the average category and did not identify them as being at high risk for falls. A time in excess of 8.5 seconds to complete the 8-Foot-Up and Go Test (Rose, Lucchese, \& Wiersma, 2006) as well as a total score on the FAB-Scale of less than 25 (Hernandez \& Rose, 2008) have been identified as cut-off scores to identify individuals at high risk for falls. The pre-assessment scores at the beginning of the study ranged from 28 to 30 on the FAB-Scale, and from 6.0 to 7.2 seconds on the 8-Foot-Up and Go Test; these results are well above the established cut-off scores. In addition to establishing the cut-off scores for individuals at high risk for falls, Hernandez and Rose (2008) also reported that for each 1-point decrease in the total FAB-Scale score the probability of falling increased by $8 \%$. Participants in the ST and BT increased their scores by an average of 1.5 points and 1.3 points respectively, whereas the SAB group increased their score by 3.4 points; there was no change in the control group's score. Even though these changes did not reach statistical significance, they nevertheless indicate a substantial reduction in the treatment groups' risks of falling, particularly in the combined SAB group. The disproportionably larger increase found in the $\mathrm{SAB}$ group is in accordance with recent reviews of literature stating that exercise as a stand-alone intervention is most effective in reducing falls if it includes a comprehensive program combining muscle strengthening as well as balance exercises (Costello \& Edelstein, 2008; Orr, Raymond, \& Fiatarone-Singh, 2008; Rose, 2008). Of course, it can also be argued that the difference in scores resulted from the disproportionably larger training effect in the $\mathrm{SAB}$ group. The $\mathrm{SAB}$ group participated in both strength training and balance training classes, receiving twice the volume of training enjoyed by the other groups.

Besides program duration and participant characteristics, differences in program design and outcome measures have been suggested as reasons for the lack of treatment effects when investigating the effectiveness of exercise interventions in fall risk reduction. Both the 8Foot-Up and Go Test as well as the FAB-Scale have been shown to be predictive measures of fall status among independently functioning older adults with test specificities of $86 \%$ and $74.6 \%$ respectively (Hernandez \& Rose, 2008; Rose, Jones, \& Lucchese, 2002). This supports the appropriateness of the outcome measures selected in the present study. With respect to program design, it can be argued that the strength training program lacked specificity since it was designed to strengthen all major muscles rather than focusing on the key muscle groups needed for balance (Orr, Raymond, \& Fiatarone-Singh, 2008). While knee extensors and flexors and hip abductors and adductors were part of the program, other key muscles groups such as ankle dorsiflexors and plantar flexors were not. The significant improvements in upper body strength for the ST and SAB group and the lack of significant changes in lower body strength can be viewed as further evidence of how the strength training program may have violated the principle of specificity. The same argument, however, cannot be made for the balance training. The BT group followed 
the FallProof ${ }^{\mathrm{TM}}$ program, which is a structured and progressive exercise program specifically designed to improve multiple dimensions of balance and mobility in older adults. Each training session is designed to enhance peripheral and central components of dynamic balance by combining multisensory, hand-eye coordination, gait pattern enhancement and variation, and strength and flexibility exercises. Taken together, these results provide evidence that there seems to be a minimal threshold in regard to the duration of exercise training to promote significant increases in dynamic balance: the six weeks of training provided by the three treatments in this study does not meet that minimum requirement.
In conclusion, the findings of the present study suggest that six weeks of strength training, balance training, or a combination of both, was not of sufficient duration to result in significant changes in dynamic balance in the present sample of community-dwelling older adults. Because a trend toward improvement was observed in all treatment groups, while the control group remained unchanged, it is tempting to speculate that a longer training period might have resulted in significant changes in dynamic balance. However, due to the study's limitations, such as the small sample size, the lack of randomization between treatment groups, and the lack of specificity in the strength training program, the above conclusion remains speculative.

\section{References}

Baechle, T., \& Earle, R. (2008). Essentials of strength training and conditioning $\left(2^{\text {nd }} e d.\right)$.

Champaign, IL: Human Kinetics.

Barnett, A., Smith, B., Lord, S., Williams, M., \& Baumand, A. (2003). Community-based group exercise improves balance and reduces falls in at-risk older people: a randomized controlled trial. Age and Ageing, 32(4), 407-14.

Benjuva, N., Melzer, L., \& Kaplanski, J. (2004). Aging-induced shifts from a reliance on sensory input to muscle cocontraction during balanced standing. The Journals of Gerontology. Series A, Biological Sciences and Medical Sciences, 59(2), M166.

Bird, M., Hill, K., \& Williams, A. (2009). Effects of resistance- and flexibility-exercise interventions on balance and related measures in older adults. Journal of Aging and Physical Activity, 17, 444-454.

Brouwer, B., Musselmann, K., \& Culham, E. (2004). Physical function and health status among seniors with and without fear of falling. Gerontology, 50, 135-141.

Campbell, A., \& Robertson, M. (2007). Rethinking individual and community fall prevention strategies: a meta-regression comparing single and multifactorial interventions. Age and Ageing, 36, 656-662.

Stevens, J., \& Sogolow, E. (2008). Preventing falls: What works A CDC compendium of effective community-based interventions from around the world. Center for Disease Control and Prevention. Retrieved from http://www.cdc.gov/HomeandRecreationalSafety/Falls/ preventfalls.html

Costello, E., \& Edelstein, J. (2008). Update on falls prevention for community-dwelling older adults: Review of single and multifactorial intervention programs. Journal of Rehabilitation Research and Development, 45(8), 1135-52.

Cumming, R., Thomas, M., Szonyi, G., Salkeld, G., O’Neill, E., Westbury, C., \& Frampton, G. (1999). Home visits by an occupational therapist for assessment and modification of environmental hazards: A randomized trial of falls prevention. Journal of the American Geriatrics Society, 47(12), 1397-1402. 
Dodd, K., Taylor, N., \& Bradley, S. (2004). Strength training for older people. M. Morris \& A. Schoo (Eds.), Optimizing exercise and physical activity in older people (pp. 125-158). Sydney, Australia: Edinburgh, Butterworth-Heinemann.

Frick, K., Kung, J., Parrish, J., \& Narrett, M. (2010). Evaluating the cost-effectivness of fall prevention programs that reduce fall-related hip fractures in older adults. Journal of the American Geriatrics Society, 58, 136-141.

Henwood, T., \& Taaffe, D. (2006). Short-term resistance training and the older adult: The effect of varied programs for the enhancement of muscle strength and functional performance. Clinical Physiology and Functional Imaging, 26(5), 305-313.

Hernandez, D., \& Rose, D. (2008). Predicting which older adults will or will not fall using the Fullerton Advanced Balance Scale. Archives of Physical Medicine and Rehabilitation, 89(12), 2309-2315.

Holmes, P. (1999). Preventing falls. Ohio State University Extension, 163(99), 1-3.

Hong, Y., Li, J., \& Robinson, P. (2000). Balance control, flexibility, and cardiorespiratory fitness among older tai chi practitioners. British Journal of Sports Medicine, 34(1), 29-34.

Huang, H., Liu, C., Huang, Y., \& Kernohan, W. (2010). Community based interventions to reduce falls among older adults in Taiwan - a long time follow-up randomized controlled study. Journal of Clinical Nursing, 19, 959-968.

Kim, S., \& Lockhart, T. (2010). Effects of 8 weeks of balance and weight training for the independently living elderly on the outcomes of induced slips. International Journal of Rehabilitation Research, 33, 49-55.

Li, F., Harmer, P., Fisher, K., \& McAuley, E. (2004). Tai chi: Improving functional balance and predicting subsequent falls in older persons. Medicine and Science in Sports and Exercise, 36(12), 2046-2052.

Lord, S., Castell, S., Corcoran, J., Dayhew, J., Matters, B., Shan, A., \& Williams, P. (2003). The effect of group exercise on physical functioning and falls in frail older people living in retirement villages: a randomized controlled trial. Journal of the American Geriatrics Society, 51(12), 1685-1692.

Merck Institute of Aging \& Health. (2004). The state of aging and health in America 2004. Retrieved July 27, 2007, from http:/www.cdc.gov/aging/pdf/State_of_Aging_and_Health_ in_America_2004.pdf

Morrison, S., Colberg, S., Mariano, M., Parson, H., \& Vinik, A. (2010). Balance training reduces falls risk in older individuals with type-2 diabetes. Diabetes Care, 33(40, 748-750.

Maurer, M., Burcham, J., \& Cheng, H. (2005). Diabetes mellitus is associated with an increased risk of falls in elderly residents of a long-term care facility. Journals of Gerontology: Series A, Biological Sciences and Medical Sciences, 60(9), 1157-1162.

Orr, R., Raymond, J., \& Fiatorone-Singh, M. (2008). Efficiency of progressive resistance training on balance performance in older adults. Sports Medicine, 88(4), 317-343.

Piirtola, M., \& Era, P. (2006). Force platform measurements as predictors of falls among older people: A review. Gerontology, 52, 1-16.

Province, M., Hadley E., Hornbrook, M., Lipsitz, L., Miller, J., Mulrow, C., Ory, M., Sattin, R., Tinetti, M., \& Wolf, S. (1995). The effects of exercise on falls in elderly patients. A preplanned meta-analysis of the FICSIT trials. Frailty and Injuries: Cooperative Studies of Intervention Techniques. The Journal of the American Medical Association, 273(17), 13411347. 
Pynoos, J. (2005). Environmental safety in the community. Presented at the National Falls Prevention Summit, Washington, D.C. Retrieved April 10, 2007, from http://www.healthy agingprograms.org/resources/FallsFree_ReviewPaper_Final.pdf

Rikli. R., \& Jones, J. (1999). Development and validation of a functional fitness test for community-residing older adults. Journal of Aging and Physical Activity, 7(2), 129-161.

Rikli, R., \& Jones, J. (2001). Senior Fitness Test Manual. Champaign, IL: Human Kinetics.

Rose, D. (2005). The role of exercise in reducing falls and fall-related injuries. Presented at the National Falls Prevention Summit, Washington, D.C. Retrieved April 10, 2007, from http://www.healthyagingprograms.org/resources/FallsFree_ReviewPaper_Final.pdf

Rose, D. (2008). Preventing falls among older adults: No "one size suits all" intervention strategy. Journal of Rehabilitation Research and Development, 45(8), 1153-1166.

Rose, D., Jones, C., \& Lucchese, N. (2002). Predicting the probability of falls in communityresiding older adults using the 8-Foot Up-and-Go: A new measure of functional mobility. Journal of Aging and Physical Activity 10(4), 466-476.

Rose, D., Lucchese, N., \& Wiersma, L. (2006). Development of a multidimensional balance scale for use with functionally independent older adults. Archives of Physical Medicine and Rehabilitation, 87(11), 1478-1485.

Rubenstein, L. (2006). Falls in older people: epidemiology, risk factors, and strategies for prevention. Age and Ageing, 35(2), 37-41.

Steadman, J., Donaldson, N., \& Kalra, L. (2003). A randomized controlled trail of an enhanced balance training program to improve mobility and reduce falls in elderly patients. Journal of the American Geriatrics Society, 51(6), 847.

Stevens, J. (2005). Falls among older adults-risk factors \& prevention strategies. Presented at the National Falls Prevention Summit, Washington, D.C. Retrieved April 10, 2007, from http://www.healthyagingprograms.org/resources/FallsFree_ReviewPaper_Final.pdf

Stevens, J., Corso, P., Finkelstein, E., \& Miller, T. (2006). The cost of fatal and non-fatal falls in older adults. Injury Prevention: Journal of the International Society and Adolescent Prevention, 12(5), 290-295.

The National Council on Aging, (2005). Falls free: Promoting a national falls prevention action plan. Retrieved April 10, 2007, from http:/www.healthyagingprograms.org/index.asp

Tinetti, M., Speechley, M., \& Ginter, S. (1988). Risk factor for falls among elderly persons living in the community. New England Journal of Medicine, 319(26), 1701.

Tinetti, M. (1994). Prevention of falls and fall injuries in elderly persons: A research agenda. Preventive Medicine, 23(5), 756-762.

Westcott, W. (2005). Strength fitness: Physiological principles and training techniques (4th ed.). Dubuque, IW: Brown and Benchmark.

Westcott, W., \& Baechle, T. (1999). Strength training for seniors: An instructor guide for developing safe and effective programs. Champaign, IL: Human Kinetics.

Wolf, S., Sattin, R., Kutner, M., O'Grady, M., Greenspan, A., \& Gregor, R. (2003). Intense tai chi exercise training and fall occurrences in older, transitional frail adults: A randomized, controlled trial. Journal of the American Geriatrics Society, 51(12), 1693-1701.

Woo, J., Hong A., Lau, E., \& Lynn, H. (2007). A randomized controlled trial of Tai Chi and resistance exercise on bone health, muscle strength, and balance in community-living elderly people. Age and Ageing, 36, 262-268. 
$\underline{\text { Author Information }}$

Petra Schuler, $\mathrm{PhD}^{*}$

Department of Health, Leisure, and Exercise Science

The University of West Florida

11000 University Parkway

Pensacola, FL 32514

Phone: (850) 474-2596

Fax: (850) 474-2106

E-mail: pschuler@uwf.edu

Christopher j. Knerl

Department of Health, Leisure, and Exercise Science The University of West Florida

Lemuel w. Taylor, PhD

Exercise Science and Sports Science,

University of Mary Hardin-Baylor

Ludmila m. Cosio-lima, $\mathrm{PhD}$

Department of Health, Leisure, and Exercise Science The University of West Florida

Karla a. Caillouet

Department of Health, Leisure, and Exercise Science The University of West Florida

* corresponding author 